\title{
Les anticorps, outils de choix pour la chirurgie guidée par fluorescence
}

en clinique depuis plusieurs années. Si l'utilisation de colorants non ciblés peut être utile dans certaines pathologies, des agents de contraste spécifiques sont indispensables en oncologie. Comme le montrent les dernières études cliniques, les anticorps monoclonaux ont toutes les caractéristiques pour jouer un rôle majeur dans ce domaine d'imagerie médicale, à condition que la cible antigénique soit pertinente. <

\section{La chirurgie guidée par la fluorescence en oncologie}

Quand elle est possible, la chirurgie est la première approche thérapeutique en oncologie et elle reste la plus efficace. De nombreuses études montrent qu'une chirurgie ayant conduit à des berges saines $(R 0)^{1}$ est le meilleur facteur pronostic dans différents types de cancers $[1,2]$. Pour le chirurgien oncologue, l'idée de voir en temps réel les zones tumorales (tumeur primaire, petites métastases, invasions dans les zones péritumorales, etc.) qu'il doit éliminer constitue donc un Graal. Cette notion de biopsie optique est en passe de devenir réalité grâce à la chirurgie guidée par la fluorescence (FGS pour fluorescence guided surgery). Cette technique de détection per opératoire imaginée il y a de nombreuses années se développe maintenant dans les blocs opératoires où les systèmes d'imagerie sont de plus en plus nombreux et, surtout, de plus en plus performants [3-6]. II y aura encore certainement des améliorations techniques, mais plusieurs dispositifs médicaux sont maintenant disponibles en clinique (par exemple, le système Spectrum et la colonne de fluorescence peropératoire SpyPhi) grâce une activité de recherche et développement très active ces dernières années $[7,8]$.

${ }^{1} \varepsilon n$ chirurgie oncologique, le terme $R$ se réfère au type de résection effectuée selon la classification suivante

R2 : Résection macroscopique incomplète

$\mathrm{Rl}$ : Atteinte microscopique de la marge de résection;

$\mathrm{RO}$ : la section chirurgicale passe à distance de la tumeur.

\author{
André Pèlegrin ${ }^{1 *}$, Marian Gutowski ${ }^{2}$, \\ Françoise Cailler ${ }^{3}$
}

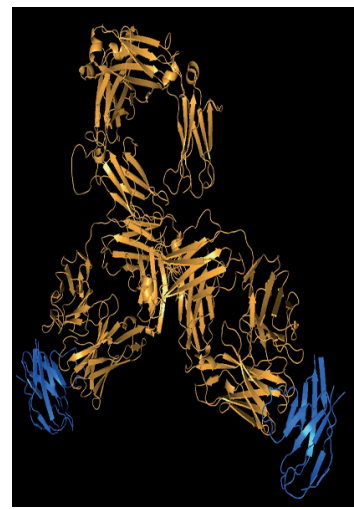

${ }^{1}$ IRCM, Institut de Recherche en Cancérologie de Montpellier, Inserm U1194, Université de Montpellier, Institut régional du Cancer de Montpellier, Montpellier, F-34298, France. ${ }^{2}$ Institut régional du Cancer de Montpellier, ICM, Montpellier, F-34298, France.

${ }^{3}$ SurgiMAb, 10 Parc Club du Millénaire, 1025 avenue Henri Becquerel, 34000 Montpellier, France.

andre.pelegrin@inserm.fr

\section{La limite actuelle : l'agent de contraste fluorescent}

Pour différentes raisons de propriétés physiques de la lumière incidente et de la fluorescence induite, et du fait des propriétés des tissus biologiques, les fluorochromes les plus pertinents pour la FGS se situent dans le proche infra-rouge (NIR, near-infrared ; 650-900 nm). Malheureusement, les colorants autorisés actuellement pour la clinique (fluorescéine, bleu de méthylène, ICG, IRD800CW) n'émettent pas dans ces longueurs d'onde et/ou sont des colorants non ciblés [9]. Ces molécules sont très utiles en ophtalmologie [10] et sont même évaluées en chirurgie oncologique, mais elles ne sont malheureusement pas les mieux adaptées [11-13]. Les chirurgiens oncologues ont besoin d'agents fluorescents NIR haute performance et ciblés sur les tumeurs pour un guidage optimal de leur geste opératoire [6]. Les agents de contraste fluorescents spécifiques des tumeurs dont deux bilans ont été faits récemment par Debie et Hernot [11] et Hernot et al. [12] peuvent se répartir en deux grandes catégories : les agents fluorescents permanents et les agents activables. Cette dernière catégorie exploite (a) certaines spécificités de l'environnement tumoral (acidité, présence d'enzymes, etc.) ou (b) certaines propriétés du colorant (extinction de fluorescence) ou de l'anticorps (internalisation) pour que la fluorescence ne soit inductible que lorsque la cible tumorale est atteinte [14]. La catégorie des agents de contraste fluorescents permanents comprend différentes molécules de ciblage: anticorps et leurs fragments, charpentes protéiques (Affibody ${ }^{\oplus}$, Nanofitin $^{\circledR}$ ), peptides et petites molécules [11]. Dans le cadre de ce numéro thématique dédié aux anticorps thérapeutiques, nous limiterons notre 
analyse aux anticorps et à leurs fragments et, plus précisément, aux molécules ayant été évaluées en clinique. En effet, les anticorps monoclonaux (AcM) et certaines molécules dérivées de ces anticorps semblent être la meilleure option pour délivrer les colorants NIR aux sites tumoraux puisque les conjugués anticorps-colorant peuvent combiner la spécificité tumorale exceptionnelle des anticorps avec des performances de fluorescence optimales de colorants dédiés [15-21]. II aura fallu plus de vingt ans pour passer de la souris à l'homme [15, 16] et des travaux des pionniers [17] aux nombreuses études cliniques actuelles, mais l'application clinique de l'immunophotodétection (IPD, c'est-à-dire l'utilisation de colorants couplés de façon covalente à des anticorps spécifiques pour la détection peropératoire des tumeurs) semble maintenant très proche [22].

\section{Les conjugués anticorps-colorants en clinique}

Les nombreuses études précliniques et quelques études cliniques concernant le développement de traceurs fluorescents pour la FGS ont fait l'objet de deux revues récentes [11, 12]. Nous ne commenterons ici que les conjugués anticorps-colorant qui sont entrés en étude clinique. En effet, si l'extrapolation des résultats des modèles précliniques à la situation chez l'homme est toujours délicate, cela est encore plus vrai en ce qui concerne la FGS en raison de l'impact de la taille de l'organisme sur les performances d'imagerie. La taille d'une souris permet de détecter quasiment tout nodule fluorescent alors que la détection de nodules profonds est un véritable défi chez l'homme. On sait en effet que le rayonnement fluorescent n'a pas la capacité de traverser des tissus de plus de 10 à $15 \mathrm{~mm}$ d'épaisseur environ.

Très logiquement, la FGS a bénéficié du développement des anticorps thérapeutiques et les premières études cliniques ont été menées avec des anticorps autorisés pour la thérapie des cancers et disponibles pour des études cliniques d'imagerie [23]. Deux cibles ont été privilégiées en raison de leur expression chez un grand nombre de patients, évitant ainsi le besoin d'une évaluation préalable de l'expression de la cible chez chaque patient: le facteur de croissance de l'endothélium vasculaire A (VEGF-A) (vascular endothelial growth factor A) et le récepteur des facteurs épidermiques de croissance (EGFR) (epidermal growth factor receptor) (Tableau I).

Deux études ont été menées avec le bévacizumab (anti-VEGF-A)IRDye800CW, l'une pour les métastases péritonéales des cancers colorectaux avec 7 patients [24] et l'autre pour les cancers du sein avec 20 patients [25]. Dans le premier cas, le bévacizumabIRDye $800 \mathrm{CW}$ a permis la détection peropératoire de lésions tumorales, mais un taux élevé de faux positifs a été rapporté par les auteurs [24]. Dans le second cas, l'utilisation de microdoses de bévacizumab-IRDye800CW, 4,5 mg pour être en-dessous du seuil de 30 nmoles $^{2}$

${ }^{2}$ Les études de microdoses sont conçues pour évaluer la pharmacocinétique ou l'imagerie de cibles spécifiques et ne sont pas conçues pour induire des effets pharmacologiques. Une microdose est définie comme étant inférieure à $1 / 100^{e}$ de la dose d'une substance d'essai calculée (d'après des données animales) pour produire un effet pharmacologique de la substance d'essai avec une dose maximale de $<100 \mu \mathrm{g}$. En raison des différences de poids moléculaire comparativement aux drogues synthétiques, la dose maximale dans les produits protéiques est $\leq 30$ nanomoles. Cela s'applique aux anticorps et correspond à une dose maximale de 4,5 mg. (https://www.fda.gov/regulatory-information/search-fda-guidance-documents/exploratory-ind-studies). défini par l'agence européenne des médicaments (EMA, European medicines agency) et la FDA (food and drug administration), n'a pas permis l'évaluation peropératoire des marges chirurgicales [25].

Sept études ont évalué le cétuximab (anti-EGFR)IRDye800CW essentiellement dans les cancers otorhino-laryngés (ORL) (37 patients au total [26-29]) et les adénocarcinomes pancréatiques (14 patients au total $[30,31])$, puis dans une étude pilote de 3 patients pour le glioblastome [32]. Une fluorescence spécifique a été observée dans une majorité des cas avec des doses injectées relativement élevées en raison de l'expression de l'EGFR dans les tissus normaux. Par exemple dans les cancers $\mathrm{ORL}$, Rosenthal et al. ont utilisé jusqu'à $62,5 \mathrm{mg} / \mathrm{m}^{2}$ de cétuximab-IRDye800CW (avec une dose de charge de $100 \mathrm{mg}$ de cétuximab non fluorescent) soit $25 \%$ de la dose thérapeutique de cétuximab [27]. De telles doses de charge ont également été utilisées dans les évaluations du panitumumab (anti-EGFR)IRDyeCW800 [29, 33-35] mais elles ont permis l'utilisation de doses de panitumumab-IRDyeCW800 plus faibles que dans le cas du cétuximab-IRDye800CW. Des doses de 0,5 à $1 \mathrm{mg} / \mathrm{kg}$ ont permis à Gao et al une détection peropératoire de tumeurs ORL [29].

Une firme de biotechnologie française a adopté une stratégie différente en choisissant un antigène cible pour ses caractéristiques adaptées à la FGS, l'antigène carcinoembryonnaire (ACE). Son expression membranaire, marquée par une densité antigénique très élevée (> 500000 molécules par cellule), est stable car l'ACE n'est pas internalisé. En outre, ce niveau d'expression reste constant quel que soit le stade tumoral (de la tumeur primaire aux métastases de tous types). L'ACE est exprimé dans pratiquement tous les cancers digestifs et dans la plupart des cancers du sein et des tumeurs pulmonaires. La présence d'ACE circulant qui est parfois citée comme problème potentiel n'en est pas un, car les patients concernés par la FGS ont, en général, un taux d'ACE circulant qui ne va pas perturber la captation tumorale d'un anticorps anti-ACE injecté par voie intra-veineuse à des doses de quelques milligrammes. $\varepsilon$ n outre, Boogerd et al. ont montré que le niveau d'ACE circulant ne reflète pas toujours le niveau d'expression de l'ACE par la tumeur, notamment chez les patients présentant une tumeur colorectale [36]. Dans cette étude, 32 patients atteints de cancer rectal sur 35 patients avaient une forte expression d'ACE au niveau tumoral. Quatorze d'entre eux présentaient un taux d'ACE circulant supérieur à la norme de $3 \mathrm{ng} / \mathrm{ml}$ mais les 18 autres étaient en dessous, démontrant qu'il n'y a pas de corrélation entre expression tumorale et taux sérique d'AcE. Pour ces raisons, plusieurs équipes s'intéressent à l'éva- 


$\left.\begin{array}{ccccc}\hline \text { Anticorps } & \text { Cible } & \text { Colorant } & \text { Cancer (indication) } & \text { Références } \\ \text { Bévacizumab } & \text { VEGF-A } & \text { IRDye800CW } & \text { Colorectal, Sein } & \text { [24, 25] } \\ \text { Cétuximab } & \text { EGFR } & \text { IRDye800CW } & \left.\begin{array}{c}\text { Tête et Cou, Pancréas, } \\ \text { Glioblastome }\end{array}\right][26-32] \\ \hline \text { Panitumumab } & \text { EGFR } & \text { IRDye800CW } & \text { Tête et Cou } & \text { Colorectal, } \\ \text { SGM-ch511 } & \text { ACE } & \text { BMl04 } & \text { Pancréas } & {[29,33-35]}\end{array}\right]$

Tableau I. Conjugués anticorps-colorant évalués en clinique pour la chirurgie guidée par fluorescence en oncologie. Adapté de Debie et Hernot [11] et de Hernot et al. [12].

luation des conjugués anti-ACE/colorant pour développer des outils pour la FGS des cancers digestifs [15-17,19-21]. Le SGM-101, constitué d'un anticorps anti-ACE couplé au colorant BM104 [37] a été évalué dans deux études cliniques chez des patients nécessitant une chirurgie pour un cancer colorectal (26 patients [38]) ou pour un cancer du pancréas (12 patients [39]). Un signal fluorescent spécifique de la tumeur a pu être observé avec une précision diagnostique de $84 \%$ des patients ayant reçu une dose de $10 \mathrm{mg}$ dans les tumeurs colorectales. Dans un groupe de 17 patients atteints de récidive ou de carcinose péritonéale de cancer colorectal et recrutés dans le cadre d'une chirurgie ouverte, la FGS a permis d'observer 19 (43\%) lésions supplémentaires par rapport à celles vues initialement par le chirurgien. Cela a entrainé une modification de l'acte chirurgical chez $32 \%$ des patients [38]. Chez les patients atteints d'adénocarcinome du pancréas, le SGM-101 a permis de détecter 21 lésions au total avec seulement un faux-positif. La présence d'un stroma desmoplastique ${ }^{3}$ caractéristique de ces tumeurs a engendré des signaux de fluorescence plus faibles que dans les cancers colorectaux. Cette étude a néanmoins montré que des anticorps peuvent tout de même atteindre et pénétrer dans ces nodules tumoraux d'adénocarcinome pancréatique [39]. Un point extrêmement intéressant pour les équipes chirurgicales est la possibilité d'éviter, grâce à la FGS, la résection d'un tissu suspect, et en fait non tumoral, notamment dans des zones où la résection inutile de tissu non tumoral entraîne une morbidité importante. Dans les cas de récidives de tumeurs rectales notamment, le chirurgien doit souvent évaluer le caractère tumoral de zones fibrotiques et cicatricielles. L'absence de fluorescence sur ces zones peut être une indication qu'il s'agit bien de tissu sain et fibrotique, et non de tissu tumoral. La bonne valeur prédictive négative de SGM-101 observée jusqu'ici [38], si elle est confirmée, permettra d'indiquer au chirurgien qu'en cas de doute, en l'absence de fluorescence, la résection d'une zone suspecte n'est pas nécessaire. En tout état de cause, SGM-101 devrait pouvoir permettre au chirurgien d'identifier les zones où une analyse histologique extemporanée doit être réalisée, ce qui se fait de façon aléatoire sans l'imagerie intraopératoire. Seuls les résultats de l'étude de phase III en cours pourront permettre de confirmer ce type de résultats (NCT03659448).

${ }^{3}$ Stroma tumoral fibro-hyperplasique dû à la production excessive de fibres de collagène qui, par endroits, se tassent et aboutissent à la formation de nappes denses, vitreuses et peu vascularisées.

\section{Les paramètres importants pour le succès de la FGS avec des anticorps fluorescents}

Avec l'ACE, nous avons vu l'importance de la densité antigénique de la cible pour l'efficacité de la FGS. En effet, si la fluorescence permet une imagerie de précision délimitant bien les nodules tumoraux qui ont fixé l'agent de contraste, elle demeure moins sensible que d'autres techniques telle que, par exemple, la médecine nucléaire. Il est donc indispensable d'utiliser une cible abondante à la surface cellulaire. Avec une densité antigénique de $10^{5}$ à $10^{6}$ molécules par cellule pour I'ACE, on peut s'attendre à obtenir une concentration de colorant allant jusqu'à $1 \mu \mathrm{M}$ en supposant un rapport 1:1 de colorant ciblé par récepteur [3, 40, 41]. Ceci correspond à la concentration maximale calculée. L'utilisation d'anticorps de haute affinité pour l'ACE comme dans le cas du SMG-101 $\left(3,21 \times 10^{-11} \mathrm{M}\right)$ devrait permettre de s'en rapprocher. Classiquement, lorsque I'on utilise des anticorps, l'expression de cette cible antigénique devra être faible voire inexistante dans les tissus sains, du moins, quand la cible est accessible à un anticorps injecté par voie intra-veineuse. Elle doit également être stable à la surface des cellules, avec une internalisation nulle ou faible, mais aussi stable entre la tumeur primaire et les éventuelles métastases et récidives loco-régionales.

L'affinité de l'anticorps pour sa cible doit être élevée, en particulier, la vitesse de dissociation (caractérisée par le $k_{\text {off }}$ ) doit être très faible pour permettre la persistance du conjugué sur la cible quand la concentration sérique diminue. Cela est indispensable pour obtenir le meilleur rapport de fluorescence entre la tumeur et les tissus environnants.

Le colorant doit avoir un rendement de fluorescence élevé car la quantité véhiculée par l'anticorps demeure faible. C'est une situation très différente de celle des colorants non-ciblés qui sont utilisés à des doses élevées pour, par exemple, la recherche d'éventuelles fuites 
vasculaires [42]. Il existe actuellement un consensus pour utiliser des colorants dans le proche infra-rouge (650-900 nm). Les deux colorants évalués en clinique dans des conjugués anticorps-colorant, IRDye800CW et BM104, ont des longueurs d'ondes d'excitation et d'émission de fluorescence différentes, respectivement, $774 / 789 \mathrm{~nm}$ et $685 / 705 \mathrm{~nm}$. Le débat entre les avantages et inconvénients de ces deux paires de longueurs d'onde n'est pas clos. La zone vers $800 \mathrm{~nm}$ permet une meilleure pénétration de la lumière incidente et une meilleure récupération de la fluorescence émise, mais elle est pénalisée par une moins bonne performance des caméras $[6,8]$. Aux environs de $700 \mathrm{~nm}$, le maximum de pénétration de la lumière dans les tissus vivants est pratiquement atteint [7] et l'utilisation d'un agent de contraste à $700 \mathrm{~nm}$ ciblé rend possible son association avec un colorant à $800 \mathrm{~nm}$ non ciblé, permettant une analyse des tissus sains ou des vaisseaux [43].

Pour le couplage du colorant à l'anticorps, la technique aléatoire reste la règle pour les conjugués anticorps-colorant étudiés jusqu'à présent en clinique. Les techniques de couplage sur des sites spécifiques développées pour les ADC devraient pouvoir s'adapter aux conjugués anticorps-colorants $[51,52](\rightarrow)$. Si les avantages théoriques sont clairs, il faudra étudier l'applicabilité de cette adaptation à grande échelle

$(\rightarrow)$ Voir les 2 articles de A. Beck et al., pages 1034 et 1043 de ce numéro et déterminer s'il y a un gain réel en clinique. En effet, la problématique est très différente en termes de toxicité entre les molécules actives très toxiques des $A D C$ et les colorants non toxiques utilisés en FGS.

Le format d'anticorps et sa taille ont un impact sur la pharmacocinétique du conjugué et sa pénétration dans la tumeur. En imagerie, il y a une tendance à utiliser des fragments d'anticorps monoclonaux, comme les fragments variables à chaîne unique (scFv) $[19,44]$ ou les nanobodies $[45,46]$, en se fondant sur l'argument qu'une cinétique courte est plus favorable et que de petites entités peuvent pénétrer plus profondément dans les nodules tumoraux. Les différentes études réalisées jusqu'à présent montrent que les conjugués préparés avec des anticorps intacts pénètrent également bien dans les tumeurs, y compris dans des tumeurs réputées difficiles d'accès telles que les adénocarcinomes du pancréas [39]. De plus, l'utilisation d'AcM intacts est compatible avec les contraintes chirurgicales. En effet, grâce à leur demi-vie plus longue (par rapport aux fragments d'anticorps), l'imagerie peropératoire à l'aide de conjugués faits avec des anticorps intacts peut être réalisée plus longtemps après l'injection et un changement de planning opératoire de quelques heures n'aura aucun impact sur la FGS. Quel que soit l'anticorps utilisé, tous les essais réalisés jusqu'à présent ont utilisé un délai de 1 à 5 jours avec, souvent, de meilleurs résultats aux temps les plus tardifs $[12,38]$. Par ailleurs, le colorant qui absorbe et émet dans le proche infrarouge est nécessairement gros et hydrophobe [6]. En utilisant des anticorps entiers, l'hydrophobicité du colorant a un effet limité sur l'hydrophilicité globale du conjugué $[15,47]$. Cela ne sera pas le cas si de petits fragments d'anticorps sont utilisés.

\section{Les paramètres à optimiser}

Jusqu'à présent les études cliniques ont essentiellement été menées avec des injections par voie intraveineuse de conjugués anticorps- colorant, mais certains auteurs préconisent une pulvérisation locale pendant l'acte chirurgical en argumentant sur la simplicité de l'approche [48]. Cependant, les composés administrés par voie topique ne peuvent mettre en évidence que les tissus et organes à la surface desquels ils ont été appliqués. Inversement, un agent de contraste injecté par voie intraveineuse peut mettre en évidence des zones plus profondes et diriger le chirurgien vers des structures qu'il n'aurait pas vues à l'œil nu [4]. Il y a donc pour l'instant un consensus parmi les experts du domaine pour dire que cette capacité à mettre en évidence les zones d'intérêt sans le biais lié à l'application locale de l'agent de contraste rend l'administration par voie intraveineuse supérieure pour les applications chirurgicales. En termes de pharmacocinétique, l'injection par voie intra-veineuse est plus favorable que l'administration topique car le délai entre l'injection et l'acte chirurgical permet la localisation du conjugué dans les nodules tumoraux et son élimination de la circulation et, par conséquent, une visualisation claire des nodules tumoraux fluorescents sans bruit de fond significatif [49].

Nous avons vu que le délai entre injection et chirurgie varie entre 1 et 5 jours pour les études cliniques des conjugués anticorps-colorants. Le délai idéal dépend certainement de la cible antigénique, de la pathologie visée et de l'anticorps utilisé. Les études cliniques en cours devront apporter des arguments concernant le meilleur délai à utiliser. Si un délai court ( 1 jour) semble plus facile à gérer en termes d'organisation hospitalière, un délai plus long (3 à 5 jours) permettra une FGS de meilleure qualité si l'anticorps utilisé est de forte affinité.

La dose optimale à injecter va également dépendre de la cible. Certaines études menées avec le cétuximab ont utilisé des doses élevées pour compenser la fixation par les organes normaux (jusqu'à $62,5 \mathrm{mg} / \mathrm{m}^{2}$; c'est-à-dire plus de $100 \mathrm{mg}$ par injection dans le premier essai clinique du cétuximab-IRDye800 contre le cancer de la tête et du cou [27]). À l'inverse, certaines études cliniques ont été menées dans un contexte de micro-dose dans lequel la dose injectée doit être inférieure à 30 nmoles $^{2}$ [25]. Des doses supérieures devront être évaluées pour connaitre la dose optimale, mais il est raisonnable de penser que des doses comprises entre 5 et $10 \mathrm{mg}$ de conjugués anticorps-colorant seront efficaces puisque des images ont été obtenues avec de telles doses avec le panitumumabIRDyeCW800 [29] et le SGM (anti-ACE)-101 [38, 39].

\section{Perspectives}

Avec de plus en plus de molécules en études cliniques $[11,12]$, dont deux en phase III (NCT03180307 pour 
I'OTL38 qui cible le récepteur du folate dans les cancers ovariens et NCT03659448 pour le SGM-101 qui cible l'ACE dans les cancers colorectaux), la chirurgie guidée par fluorescence avec des agents de contraste ciblés est clairement arrivée à maturité. Les résultats de ces études de phase III auront un impact majeur sur le développement de la FGS. En l'absence de technique concurrente actuelle, la définition des objectifs de ces études, qui permettraient d'autoriser une mise sur le marché de ces molécules si ceux-là sont atteints, est délicate. Elle fait l'objet de groupes de travail auxquels participent spécialistes du domaine et agences réglementaires, dont la FDA [50].

Un point majeur est l'enthousiasme des chirurgiens pour cette nouvelle pratique. Il est vrai que cette idée de «biopsie optique » permettant de voir les zones nécessitant une exérèse a été envisagée depuis longtemps et la FGS la rend enfin possible. Comme toute technique, la FGS nécessite un apprentissage. II faudra être attentif à la formation des équipes chirurgicales lors du déploiement de la technique mais, connaissant la facilité avec laquelle les chirurgiens ont accepté les robots chirurgicaux tels que le Da Vinci, nous pouvons être confiants. $\diamond$

\section{SUMMARY}

Antibodies, tools of choice for fluorescence-guided surgery

Fluorescence-guided surgery has been developing in clinics for several years. While the use of non-targeted dyes may be useful in certain diseases, specific contrast agents are essential in oncology. As shown in the latest clinical studies, monoclonal antibodies have all the characteristics to play a major role in this field of medical imaging, provided the antigenic target is relevant. $\diamond$

\section{LIENS D'INTÉRÊT}

FC est Présidente de SurgimAb qui développe le SMG-101.

$F C, A P, M G$ sont co-fondateurs et actionnaires de SurgiMAb.

\section{RÉFÉRENCES}

1. Wong LS, McMahon J, Devine J, et al. Influence of close resection margins on local recurrence and disease-specific survival in oral and oropharyngeal carcinoma. Br J Oral Maxillofac Surg 2012 ; $50: 102-8$.

2. Vos EL, Gaal J, Verhoef C, et al. Focally positive margins in breast conserving surgery: predictors, residual disease, and local recurrence. Eur J Surg Oncol 2017 ; 43 : 1846-54.

3. Vahrmeijer AL, Hutteman M, Vorst JR van der, et al. Image-guided cancer surgery using nearinfrared fluorescence. Nat Rev Clin Oncol 2013; 10 : 507-18.

4. Nguyen $\mathrm{QT}, \mathrm{Tsien} \mathrm{Ry}$. Fluorescence-guided surgery with live molecular navigation: a new cutting edge. Nat Rev Cancer $2013 ; 13: 653-62$.

5. Rosenthal EL, Warram JM, Boer $\varepsilon$ de, et al. Successful translation of fluorescence navigation during oncologic surgery: a consensus report. J Nucl Med 2016 ; 57 : 144-50.

6. Gioux S, Choi HS, Frangioni JV. Image-guided surgery using invisible near-infrared light: fundamentals of clinical translation. Mol lmaging $2010 ; 9: 237-55$.

7. Keereweer S, Van Driel PBAA, Snoeks TJA, et al. Optical image-guided cancer surgery: challenges and limitations. Clin Cancer Res $2013 ; 19: 3745-54$

8. DSouza AV, Lin H, Henderson $\varepsilon R$, et al. Review of fluorescence guided surgery systems: identification of key performance capabilities beyond indocyanine green imaging. J Biomed Opt $2016 ; 21: 80901$.

9. van Dam GM, Themelis G, Crane LMA, et al. Intraoperative tumor-specific fluorescence imaging in ovarian cancer by folate receptor- $\alpha$ targeting: first in-human results. Nat Med $2011 ; 17: 1315-9$.

10. Reinhart MB, Huntington CR, Blair LJ, et al. Indocyanine green: historical context, current applications, and future considerations. Surg Innov $2016 ; 23: 166-75$.

11. Debie $P$, Hernot $S$. Emerging fluorescent molecular tracers to guide intra-operative surgical decision-making. Front Pharmacol 2019; $10: 510$.

12. Hernot $S$, Manen $L$ van, Debie $P$, et al. Latest developments in molecular tracers for fluorescence image-guided cancer surgery. Lancet Oncol 2019 ; 20 : e354-67.
13. Liberale G, Bourgeois P, Larsimont D, et al. Indocyanine green fluorescenceguided surgery after IV injection in metastatic colorectal cancer: a systematic review. Eur J Surg Oncol 2017 ; 43 : 1656-67.

14. Mochida A, Ogata F, Nagaya T, et al. Activatable fluorescent probes in fluorescence-guided surgery: practical considerations. Bioorg Med Chem $2018 ; 26: 925-30$.

15. Pèlegrin A, Folli S, Buchegger F, et al. Antibody-fluorescein conjugates for photoimmunodiagnosis of human colon carcinoma in nude mice. Cancer $1991 ; 67: 2529-37$.

16. Folli S, Westermann P, Braichotte D, et al. Antibody-indocyanin conjugates for immunophotodetection of human squamous cell carcinoma in nude mice. Cancer Res $1994 ; 54: 2643-9$.

17. Folli $S$, Wagnières $G$, Pèlegrin $A$, et al. Immunophotodiagnosis of colon carcinomas in patients injected with fluoresceinated chimeric antibodies against carcinoembryonic antigen. Proc Natl Acad Sci USA $1992 ; 89$ : 7973-7.

18. Korb ML, Hartman YE, Kovar J, et al. Use of monoclonal antibodyIRDye800CW bioconjugates in the resection of breast cancer. J Surg Res $2014 ; 188$ : 119-28.

19. Boonstra MC, Tolner B, Schaafsma BE, et al. Preclinical evaluation of a novel CEA-targeting near-infrared fluorescent tracer delineating colorectal and pancreatic tumors. Int J Cancer 2015 ; 137 : 1910-20.

20. Metildi CA, Kaushal S, Snyder CS, et al. Fluorescence-guided surgery of human colon cancer increases complete resection resulting in cures in an orthotopic nude mouse model. J Surg Res 2013; 179: 87-93.

21. Metildi CA, Kaushal S, Pu M, et al. Fluorescence-guided surgery with a fluorophore-conjugated antibody to carcinoembryonic antigen (CEA), that highlights the tumor, improves surgical resection and increases survival in orthotopic mouse models of human pancreatic cancer. Ann Surg Oncol $2014 ; 21: 1405-11$.

22. Tipirneni KE, Warram JM, Moore LS, et al. Oncologic procedures amenable to fluorescence-guided surgery. Ann Surg $2017 ; 266$ : 36-47.

23. Cohen R, Stammes MA, de Roos IH, et al. Inert coupling of IRDye $800 \mathrm{CW}$ to monoclonal antibodies for clinical optical imaging of tumor targets. EJNMMI Res $2011 ; 1: 31$.

24. Harlaar NJ, Koller M, de Jongh SJ, et al. Molecular fluorescence-guided surgery of peritoneal carcinomatosis of colorectal origin: a single-centre feasibility study. Lancet Gastroenterol Hepatol 2016 ; 1 : 283-90.

25. Lamberts $L \varepsilon$, Koch $M$, de Jong JS, et al. Tumor-specific uptake of fluorescent bevacizumab-IRDye $800 \mathrm{CW}$ microdosing in patients with primary breast cancer: a Phase I feasibility study. Clin Cancer Res $2017 ; 23: 2730-41$.

26. de Boer $\varepsilon$, Warram JM, Tucker MD, et al. In vivo fluorescence immunohistochemistry: localization of fluorescently labeled cetuximab in squamous cell carcinomas. Sci Rep $2015 ; 5$ : 10169.

27. Rosenthal $\varepsilon L$, Warram JM, de Boer $\varepsilon$, et al. Safety and tumor specificity of cetuximab-IRDye 800 for surgical navigation in head and neck cancer. Clin Cancer Res $2015 ; 21: 3658-66$.

28. Rosenthal EL, Moore LS, Tipirneni K, et al. Sensitivity and specificity of cetuximab-IRDye800CW to identify regional metastatic disease in head and neck cancer. Clin Cancer Res $2017 ; 23: 4744-52$.

29. Gao RW, Teraphongphom N, de Boer $\varepsilon$, et al. Safety of panitumumabIRDye $800 \mathrm{CW}$ and cetuximab-IRDye $800 \mathrm{CW}$ for fluorescence-guided surgical navigation in head and neck cancers. Theranostics $2018 ; 8: 2488-95$.

30. Tummers WS, Miller SE, Teraphongphom NT, et al. Intraoperative pancreatic cancer detection using tumor-specific multimodality molecular imaging. Ann Surg Oncol $2018 ; 25: 1880-8$.

31. Tummers WS, Miller SE, Teraphongphom NT, et al. Detection of visually occult metastatic lymph nodes using molecularly targeted fluorescent imaging during surgical resection of pancreatic cancer. HPB (Oxford) $2019 ; 21$ : 883-90.

32. Miller SE, Tummers WS, Teraphongphom N, et al. First-in-human intraoperative near-infrared fluorescence imaging of glioblastoma using cetuximab-IRDye800. J Neurooncol 2018 ; 139: 135-43.

33. Keulen $\mathrm{S}$ van, Berg NS van den, Nishio N, et al. Rapid, non-invasive fluorescence margin assessment: Optical specimen mapping in oral squamous cell carcinoma. Oral Oncol $2019 ; 88: 58-65$.

34. Keulen S van, Nishio N, Fakurnejad S, et al. The clinical application of fluorescence-guided surgery in head and neck cancer. J Nucl Med 2019; 60 : 758-63.

35. Gao RW, Teraphongphom NT, van den Berg NS, et al. Determination of tumor margins with surgical specimen mapping using near-infrared fluorescence. Cancer Res 2018 ; 78 : 5144-54. 


\section{RÉFÉRENCES}

36. Boogerd LSF, Vuijk FA, Hoogstins CES, et al. Correlation between preoperative serum carcinoembryonic antigen levels and expression on pancreatic and rectal cancer tissue. Biomark Cancer $2017 ; 9$ : 1179299X17710016.

37. Gutowski M, Framery B, Boonstra MC, et al. SGM-101: an innovative near-infrared dye-antibody conjugate that targets CEA for fluorescence-guided surgery. Surg Oncol $2017 ; 26$ : 153-62.

38. Boogerd LSF, Hoogstins CES, Schaap DP, et al. Safety and effectiveness of SGM-101, a fluorescent antibody targeting carcinoembryonic antigen, for intraoperative detection of colorectal cancer: a dose-escalation pilot study. Lancet Gastroenterol Hepatol 2018 ; 3 : 181-91.

39. Hoogstins CES, Boogerd LSF, Sibinga Mulder BG, et al. Image-guided surgery in patients with pancreatic cancer: First results of a clinical trial using SGM-101, a novel carcinoembryonic antigen-targeting, near-infrared fluorescent agent. Ann Surg Oncol $2018 ; 25: 3350-7$.

40. Boonstra MC, de Geus SWL, Prevoo HAJM, et al. Selecting targets for tumor imaging: an overview of cancer-associated membrane proteins. Biomark Cancer 2016; $8: 119-33$.

41. Frangioni JV. New technologies for human cancer imaging. J Clin Oncol $2008 ; 26: 4012-21$

42. Boni L, David G, Mangano A, et al. Clinical applications of indocyanine green (ICG) enhanced fluorescence in laparoscopic surgery. Surg Endosc $2015 ; 29$ : 2046-55.

43. Owens $\varepsilon A$, Henary $M$, $\varepsilon l$ Fakhri $G$, et al. Tissue-specific near-infrared fluorescence imaging. Acc Chem Res $2016 ; 49$ : 1731-40.

44. Ortega C, Herbet A, Richard S, et al. High level prokaryotic expression of anti-Müllerian inhibiting substance type II receptor diabody, a new recombinant antibody for in vivo ovarian cancer imaging. J Immunol Methods $2012 ; 387$ : 11-20.

45. Driel PBAA van, Vorst JR van der, Verbeek FPR, et al. Intraoperative fluorescence delineation of head and neck cancer with a fluorescent anti-epidermal growth factor receptor nanobody. Int J Cancer $2014 ; 134: 2663-73$
46. Krüwel T, Nevoltris D, Bode J, et al. In vivo detection of small tumour lesions by multi-pinhole SPECT applying a $(99 \mathrm{~m})$ Tc-labelled nanobody targeting the epidermal growth factor receptor. Sci Rep $2016 ; 6: 21834$.

47. Sato K, Gorka AP, Nagaya T, et al. Effect of charge localization on the in vivo optical imaging properties of near-infrared cyanine dye/monoclonal antibody conjugates. Mol Biosyst 2016; 12 : 3046-56.

48. Urano Y, Sakabe M, Kosaka N, et al. Rapid cancer detection by topically spraying a $\gamma$-glutamyltranspeptidase-activated fluorescent probe. $\mathrm{Sci}$ Transl Med $2011 ; 3: 110$ rallo.

49. Cuesta AM, Sainz-Pastor N, Bonet J, et al. Multivalent antibodies: when design surpasses evolution. Trends Biotechnol $2010 ; 28: 355-62$

50. Tummers WS, Warram JM, Tipirneni KE, et al. Regulatory aspects of optical methods and exogenous targets for cancer detection. Cancer Res 2017 ; 77 2197-206.

51. Beck A, Dumontet C, Joubert N. Les immunoconjugués en oncologie : les raisons du succès récent d'une approche ancienne. Med Sci (Paris) 2019 ; $35: 1034-42$

52. Beck $A$, Dumontet $C$, Joubert N. Les immunoconjugués en oncologie : les nouvelles stratégies en développement. Med Sci (Paris) 2019; 35 : 1043-53.

TIRÉS À PART

A. Pèlegrin

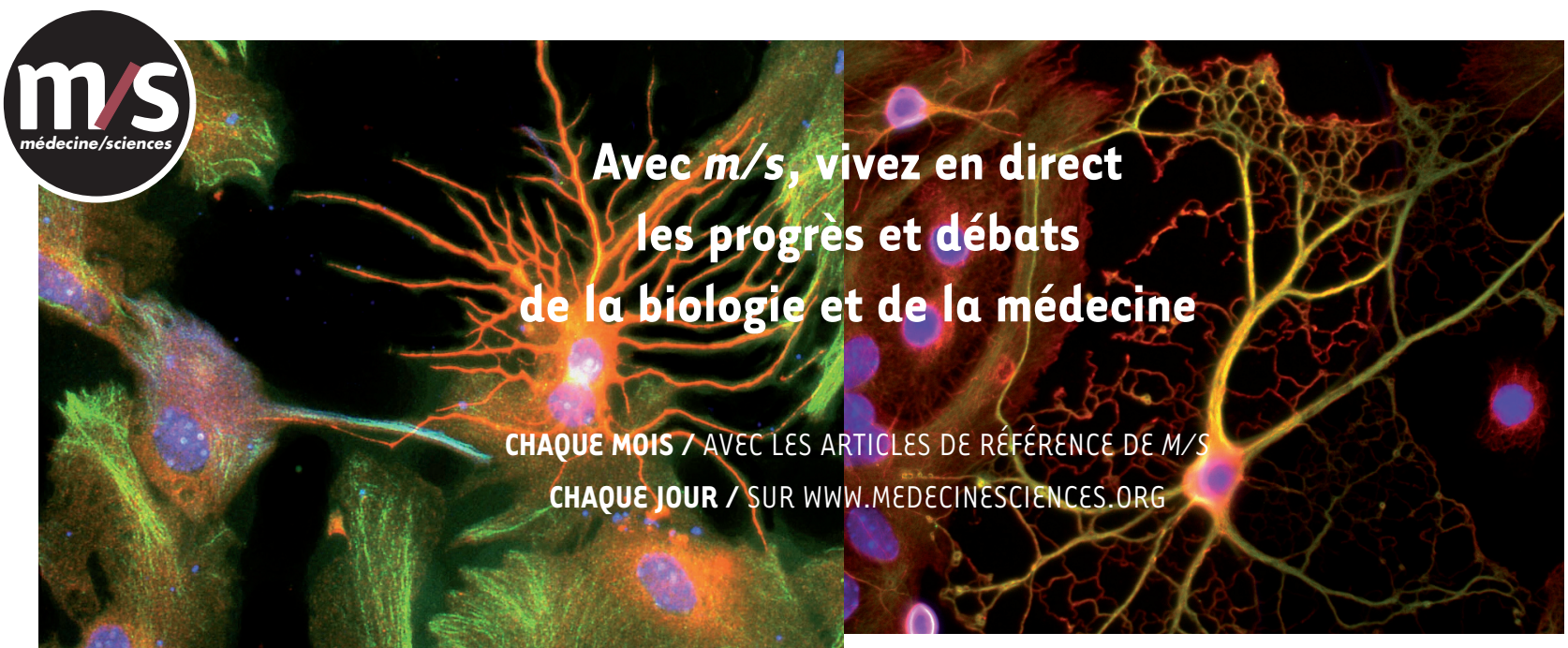

Abonnez-vous sur

wWw.medecinesciences.org

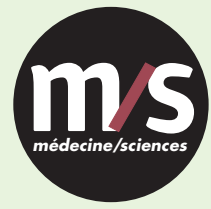

Tarifs d'abonnement m/s - 2019

Abonnez-vous

à médecine/sciences
> Grâce à $m / s$, vivez en direct les progrès des sciences biologiques et médicales

Bulletin d'abonnement page 1230 dans ce numéro de $\mathrm{m} / \mathrm{s}$

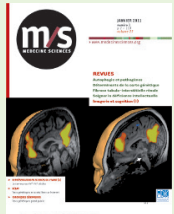

